\title{
CHOOSING SMALL WEIGHTS FOR MULTIPLE ERROR DETECTION
}

\author{
RICHARD P. BRENT, F. T. LUK, AND C. J. ANFINSON
}

\begin{abstract}
The weighted checksum technique has been demonstrated to be effective in multiple error detection. It has been shown that, in order to guarantee error detection, the chosen weight vectors must satisfy some very specific properties regarding linear independence. Previous weight generating methods that fulfil the independence criteria have problems with numerical overflow. We present a new scheme that generates weight vectors to meet the requirements of independence and to avoid the difficulties with overflow.
\end{abstract}

\section{Comments}

Only the Abstract is given here. The full paper appeared as [2], and a revision appeared as [1]. The code constructed is a BCH code, and the proof of Proposition 1 is similar to that of Theorem 6.6 .2 of [3].

\section{REFERENCES}

[1] R. P. Brent, F. T. Luk and C. J. Anfinson, "Checksum schemes for fault tolerant systolic computing", Mathematics in Signal Processing II (edited by J. G. McWhirter), Clarendon Press, Oxford, 1990, 791-804. rpb112.

[2] R. P. Brent, F. T. Luk and C. J. Anfinson, "Choosing small weights for multiple error detection", Proceedings SPIE, Volume 1058, High Speed Computing II, SPIE, Los Angeles, 1989, 130-136. rpb114.

[3] J. H. van Lint, Introduction to Coding Theory, Graduate Texts in Mathematics 86, Springer-Verlag, 1982.

(Brent) Computer Sciences Lab, Australian National University, Canberra

(Luk) School of Electrical Engineering, Cornell University, Ithaca, New York

(Anfinson) Center for Applied Mathematics, Cornell University, Ithaca, New York

1991 Mathematics Subject Classification. Primary 94B05; Secondary 68Q35, 94B35, 94B99.

Key words and phrases. Error correction, error detection, fault tolerance, algorithmic fault tolerance, ReedSolomon code, BCH code, overflow.

Copyright (c) 1989, SPIE..

Comments (c) 1993, R. P. Brent. $\quad$ rpb114a typeset using $\mathcal{A M}_{\mathcal{M}} \mathcal{S}$ - $\mathrm{AT}_{\mathrm{E}} \mathrm{X}$. 